\title{
Radial Velocities as an Exoplanet Discovery Method
}

\author{
Jason T. Wright
}

\begin{abstract}
The precise radial velocity technique is a cornerstone of exoplanetary astronomy. Astronomers measure Doppler shifts in the star's spectral features, which track the line-of-sight gravitational accelerations of a star caused by the planets orbiting it. The method has its roots in binary star astronomy, and exoplanet detection represents the low-companion-mass limit of that application. This limit requires control of several effects of much greater magnitude than the signal sought: the motion of the telescope must be subtracted, the instrument must be calibrated, and spurious Doppler shifts "jitter" must be mitigated or corrected. Two primary forms of instrumental calibration are the stable spectrograph and absorption cell methods, the former being the path taken for the next generation of spectrographs. Spurious, apparent Doppler shifts due to non-center-of-mass motion (jitter) can be the result of stellar magnetic activity or photospheric motions and granulation. Several avoidance, mitigation, and correction strategies exist, including careful analysis of line shapes and radial velocity wavelength dependence.
\end{abstract}

\section{Radial Velocities in Astronomy}

Most of what we know about heavens comes from information encoded in various forms of light. While many important advances have come from studies of meteorites, in situ measurements of the Solar System by space probes, detection of high energy particles, and more recently by observations of astronomical neutrinos and gravitational waves; the vast majority of astronomical observations come from eking as much information as possible from photons.

Because of this restriction, certain properties of stars and galaxies are much more easily determined than others. Positions on the sky can be measured to great accu-

Department of Astronomy \& Astrophysics and Center for Exoplanets and Habitable Worlds 525 Davey Laboratory The Pennsylvania State University University Park, PA, 16802, USA e-mail: astrowrightegmail.com 
racy, but distances can often only be approximated. For the time derivatives of these quantities, the situation is usually reversed; motions of objects in the plane of the sky can be imperceptible due to the vast distances involved, but thanks to the Doppler shift motions along the line of sight (radial velocities) can often be measured with great precision.

Much of astronomy is concerned with the motions of objects under the influence of their mutual gravity, and so radial velocity measurements naturally form a cornerstone of astronomical research. They tell us the masses of everything from moons in the solar system to stars to galaxies; they have revealed the existence of unseen planets, dark matter, and the expansion and acceleration of the universe itself.

\section{Binary Systems}

Binary stars provide much of our foundational knowledge of stellar structure. The relative masses of the stars in a binary system can be determined from the stars' orbits about their common center of mass from the ratio of the amplitudes of their radial velocity variations. Further knowledge of the non-radial component of their motion - either from astrometric measurements of their orbits or from the fact that they eclipse each other - yields absolute masses for the individual stars from Newton's Laws.

In some cases, only a single star in a binary system is bright enough to have its spectrum measured (a single-lined spectroscopic binary system, or SB1). In this case, the (semi-)amplitude of the radial velocity variations $K$ over the course of an orbit with period $P$ is related to the properties of the system via the so-called mass function, $f$ :

$$
f \equiv \frac{P K^{3}\left(1-e^{2}\right)^{\frac{3}{2}}}{2 \pi G}=\frac{M_{\text {unseen }}^{3} \sin ^{3} i}{\left(M_{\text {unseen }}+M_{\text {seen }}\right)^{2}}
$$

Here, $e$ represents the eccentricity of the orbit, $i$ represents the inclination of the plane of the orbit with respect to the plane of the sky $(i=0$ represents a face-on orbit with no radial component), $G$ is Newton's constant, and $M_{\text {seen }}$ and $M_{\text {unseen }}$ are the masses of the seen and unseen stars. The phase and orientation of the elliptical orbit with respect to the line-of-sight are represented by an additional pair of parameters, $T_{0}$ and $\omega$, and the overall radial velocity of the center of mass of the system is usually given by $\gamma$. The orientation of the orbit on the plane of the sky is given by $\Omega$ but, as with $i$, its measurement requires additional information not found in the radial velocities. (For a more detailed discussion of single-lined spectroscopic orbits in general, see Wright and Gaudi (2013), and for a more detailed treatment of the mechanics of translating measurements into orbital parameters see Wright and Howard (2009).)

The orbital parameters measured in this way represent the orbit of the observed star about the center of mass of the system; in single-lined system the existence 
of the unseen object is often inferred from this motion, and its (unmeasured and usually unreported) orbital elements are identical except that $K$ is scaled by a factor of $M_{\text {unseen }} / M_{\text {seen }}$ and $\omega$ differs by $\pi$.

This application of radial velocities is not restricted to binary stars; it is used to detect unseen binary companions ranging in mass from black holes to planets. For the case of exoplanets, we can approximate the mass function in the large mass-ratio limit $M_{\text {unseen }} / M_{\text {seen }}=M_{\text {planet }} / M_{*} \ll 1$, yielding the more familiar equation relating the amplitude of a Doppler shift to the mass and orbital properties of the planet:

$$
K \approx\left(\frac{2 \pi G}{P M_{*}^{2}}\right)^{\frac{1}{3}} \frac{M_{\text {planet }} \sin i}{\sqrt{1-e^{2}}}
$$

The ability to detect exoplanets with precise radial velocimetry thus depends on the planet's mass, inclination, and orbital period (there is also a dependence on orbital eccentricity, which is complex but weak for low eccentricities). The period dependence is weak, and for giant planets, detectability is often limited more by the duration of the observations than their RV amplitude.

The strongest dependence is on the quantity $M_{\text {planet }} \sin i$ (pronounced "em-sineeye"). The true mass of the planet is larger by a geometric factor $1 / \sin i$, and so this is often referred to as the "minimum mass" of the planet (a precise term which can be properly computed from the more exact Equation 11. For scale, we can express the quantities in more familiar units:

$$
\begin{aligned}
K & =28 \mathrm{~m} / \mathrm{s}\left(1-e^{2}\right)^{-\frac{1}{2}}(P / \mathrm{yr})^{\frac{1}{3}}\left(M_{*} / M_{\odot}\right)^{-\frac{2}{3}}\left(M_{\text {planet }} / M_{\text {Jupiter }}\right) \sin i \\
& =200 \mathrm{~m} / \mathrm{s}\left(1-e^{2}\right)^{-\frac{1}{2}}(P / \text { day })^{\frac{1}{3}}\left(M_{*} / M_{\odot}\right)^{-\frac{2}{3}}\left(M_{\text {planet }} / M_{\text {Jupiter }}\right) \sin i \\
& =0.09 \mathrm{~m} / \mathrm{s}\left(1-e^{2}\right)^{-\frac{1}{2}}(P / \mathrm{yr})^{\frac{1}{3}}\left(M_{*} / M_{\odot}\right)^{-\frac{2}{3}}\left(M_{\text {planet }} / M_{\text {Earth }}\right) \sin i \\
& =0.64 \mathrm{~m} / \mathrm{s}\left(1-e^{2}\right)^{-\frac{1}{2}}(P / \text { day })^{\frac{1}{3}}\left(M_{*} / M_{\odot}\right)^{-\frac{2}{3}}\left(M_{\text {planet }} / M_{\text {Earth }}\right) \sin i
\end{aligned}
$$

We see here why the first strong exoplanet detections (Latham et al 1989; Mayor and Queloz 1995) were of Jovian planets in short period orbits: 51 Peg $b$, for instance, has minimum mass of $0.4 M_{\text {Jupiter }}$ and a 4.2 day orbit, so its RV amplitude is a relatively large $60 \mathrm{~m} / \mathrm{s}$. Jupiter induces a $12 \mathrm{~m} / \mathrm{s}$ amplitude motion on the Sun; the Earth's motion is a (currently) undetectable $9 \mathrm{~cm} / \mathrm{s}$.

Finally, the dependence on stellar mass means that exoplanet detection is in principle most sensitive around the lowest mass stars, a point we shall revisit later.

\section{Redshift Measurements}

Starlight is imprinted with many absorption lines by ions, atoms, and molecules in stellar atmospheres, and atomic physics allows us to calculate or measure the rest wavelengths $\lambda_{\text {rest }}$ of these lines. A star's radial motion causes these lines to 
be Doppler shifted to their observed wavelengths $\lambda_{\text {obs }}$. The Doppler formula then allows astronomers to calculate the relative radial speed between the star and the observatory that measured the light $v_{r}$ via the redshift $z$ :

$$
z \equiv \frac{\lambda_{\text {obs }}-\lambda_{\text {rest }}}{\lambda_{\text {rest }}}=\frac{1}{\gamma\left(1+v_{r} / c\right)}-1
$$

where here (unlike above) $\gamma$ is the relativistic factor $1 /\left(1-(v / c)^{2}\right), c$ is the speed of light, and $v$ is the scalar relative speed between the frame of the star and the observatory (which is not necessarily in the radial direction).

Radial velocity measurements made with respect to the "laboratory" in this manner are called absolute radial velocities, and form the basis of our understanding of the dynamics of the Galaxy and the expansion of the Universe. Such measurements have accuracy limited by the wavelength calibration of the spectrograph and understanding of complicating factors such as the internal motions of the emitting material and redshifts from General Relativity. Typical accuracies of absolute radial velocities of stars are of order $100 \mathrm{~m} / \mathrm{s}$ (Chubak et al 2012).

More precise measurements can be made by measuring differential radial velocities, that is, the change in the redshift between two epochs. Differential measurements have the advantage that some uncertainties (such as those from systematic effects, imperfectly known rest wavelengths, or the model of the emitting material) will effect all measurements made with a certain instrument or technique equally, and so differences between measurements do not suffer from them. Thus, measurements of the change in the redshift of a star's spectral features can be made to more than two orders of magnitude better precision than the accuracy of the absolute redshift.

\section{Barycentric Motion}

The observatory measuring a radial velocity is on a moving platform: the Earth. The Earth rotates at $\sim 300 \mathrm{~m} / \mathrm{s}$ and orbits the Sun at $\sim 30 \mathrm{~km} / \mathrm{s}$. As a result, the measured radial velocity of a perfectly stable star will appear to vary on diurnal and annual timescales by some fraction of these amounts. Correcting measurements for this motion is known as a barycentric correction, where the term barycenter refers to the center of mass of the Solar System. The idea is that one must correct measurements made on the Earth to measurements that would have been made in the (inertial) frame comoving with the Solar System's center of mass.

These motions are often much larger than the orbital motions of the stars, and in the case of stars moving under the influence of planets the barycentric correction can be four or five orders of magnitude larger. Thus, practically speaking, the problem of radial velocity precision is not one of measuring very small redshifts, but one of measuring modest redshifts very precisely, often to one part in $10^{4}$ or better. 
Fortunately, the motion of the Earth is well studied and well measured for purposes that require much more precision than exoplanet detection. Ephemerides (tables describing the location of celestial bodies as a function of time, pronounced eff-em-AIR-i-deez, singular ephemeris, pronounced eh-FEM-er-iss) for the Earth in the barycentric frame are maintained by the Jet Propulsion Laboratory and others, and the orientation of the Earth can be predicted into the future with high accuracy (and measurements of that orientation are available through, for instance, the International Earth Rotation Service). Finally, all modern observatories provide precise timekeeping so observations can be tagged with an appropriate time stamp for barycentric correction algorithms later. A detailed description of the barycentric correction process in the context of exoplanet detection can be found in Wright and Eastman (2014).

\section{Measuring Precise Radial Velocities}

Two primary methods of precise radial velocimetry have been used to discover and characterize exoplanets via the reflex velocities of their host stars: absorption cell spectroscopy and spectrograph stabilization. They differ primarily in the method used to calibrate the spectrograph.

The chief limit to the precision of differential redshift measurements is the wavelength calibration of the spectrograph. Typical RV spectrographs resolve starlight with a power of $R=\lambda / \Delta \lambda \sim 50,000-100,000$, meaning that a shift of a single pixel corresponds to a change in radial velocity of $1 \mathrm{~km} / \mathrm{s}$. Since giant planets change their host stars's velocities by of order tens of $\mathrm{m} / \mathrm{s}$, one must measure shifts to a precision of $10^{-2}$ pixel for giant planets and two or three orders of magnitude better than that to detect Earth-mass planets. Since typical pixels on astronomical detectors are of order $15 \mu \mathrm{m}$ across, one is measuring the "motions" of stellar lines to a fraction of a nanometer.

Astronomical spectrographs are not this stable. Most are general-purpose instruments with many moving parts that are actuated so the spectrograph can be used for many purposes. A given wavelength of light cannot typically be expected to land to its usual position by much better than a pixel from night to night (or year to year). The solution then is to employ some combination of stabilization and calibration and employ differential techniques.

Wright and Gaudi (2013) provide a discussion of the historical development and first applications of both techniques, and Fischer et al (2016) provides an overview of the state of the field. 


\section{Stable spectrographs}

The first strong exoplanet detection (recognized as such only after the fact) was that of Latham et al (1989), who stabilized a spectrograph in two ways: by removing it from the telescope (to prevent its orientation with respect to the local gravity vector from changing, minimizing flexure) and coupling it to the starlight via an optical fiber (which served to "scramble" the starlight, presenting the spectrograph with a uniform image of the star despite variations in guiding and seeing). Remaining variations in the wavelength solution of the spectrograph (from thermal changes to the spectrograph or small variations in the fiber illumination) were tracked with a thorium-argon emission lamp, which provided a stable set of reference lines. This combination of stabilization and calibration allowed for precise differential measurements over the course of several nights

Since then, this technique has been taken to extreme lengths. State-of-the-art stable radial velocimeters today control the vibration, temperature, and pressure of spectrographs with exquisite precision using cryostats and vacuum chambers. The remaining, unavoidable changes in the spectrograph (from, for instance, slow changes in the crystalline structure of the metals involved or irregular thermal outputs from the detector electronics) are tracked via emission sources such as laser frequency combs, which are locked to atomic clocks and provide essentially perfect wavelength references.

Today, the state of the art is represented by the HARPS (Queloz et al 2001b) and ESPRESSO (Pepe et al 2010) spectrographs of ESO, which are stable below the 1 $\mathrm{m} / \mathrm{s}$ level (the latter aspires to $10 \mathrm{~cm} / \mathrm{s}$ precision.)

\section{Absorption cell spectroscopy}

A more widely applicable method, and the one responsible for most of the first several dozen exoplanet discoveries, is that of absorption cell calibration. A cell of gas is placed in the path of the starlight, imprinting it with a set of spectral absorption features. These features follow the starlight through the spectrograph, and so suffer all of the same instrumental shifts as the starlight itself. They thus provide an opportunity to track and calibrate all changes in a spectrograph, no matter how unstable.

Early work by Campbell and Walker (1979) used HF gas and achieved precision near $10 \mathrm{~m} / \mathrm{s}$. Later, Marcy and Butler (1992) found success with molecular iodine $\left(\mathrm{I}_{2}\right)$, and Butler et al (1996) demonstrated precision near $3 \mathrm{~m} / \mathrm{s}$ through careful modeling of the iodine spectrum and the spectrograph line spread function. Many highresolution spectrographs have since been retrofitted with absorption cells, turning them into Doppler velocimeters capable of $1-10 \mathrm{~m} / \mathrm{s}$ precision. 


\section{Radial Velocity Jitter: Spurious Doppler Signals}

Observed stellar spectroscopic absorption features are the product of absorption, emission, scattering, and motions of gasses throughout stellar atmospheres, across the differentially rotating stellar disk. The lines will change their shape and centroid positions due to many effects besides a true center-of-mass movement of the star itself. This effect is called jitter and it operates on a variety of timescales, amplitudes, and with a range of noise distributions.

The problem is most severe for spotty, rapidly rotating, and low-gravity stars. This not only makes detection sensitivity progressively worse for younger and more evolved stars, but also introduces the more insidious problem of false detections around these stars, a problem that has plagued the field since its inception (e.g. Queloz et al 2001a).

The solution to the problem of detecting planets with Doppler amplitudes near or below the level of the jitter requires a variety of avoidance and mitigation techniques.

\section{Stellar Magnetic Activity}

Cool stars-those with convective envelopes and enough spectral features that precise Doppler work is possible - have dynamos that generate surface magnetic fields. These fields interact strongly with the stellar atmosphere and wind, which are (at least partly) ionized, and affect their motions.

The wind in particular is heavily influenced by the field, which is anchored to the rotating star, and this coupling causes the wind to carry angular momentum away from the star, which thus spins down. This spin-down weakens the dynamo, lessening the effects of activity-based jitter. Young stars thus spin quickly and have a lot of activity-based jitter, and the problem is not as severe for older stars.

On the surface of stars, magnetic fields cause surface brightness inhomogeneities, including bright plage and faculae, and dark spots. This breaks the symmetry of the rotational broadening kernel of the emergent intensity from the stellar atmosphere, producing asymmetric line profiles. That is, a bright spot on the approaching limb of the star will produce a line with a slightly blueshifted centroid with respect to a homogeneous stellar surface, and a spot there will produce a redshifted line. This effect is proportional to the spot contrast and surface coverage, and also the projected rotational velocity of the star, both of which are worse for younger stars.

\section{Photospheric Motions and Global Oscillations}

Cool stars have surfaces characterized by granulation-a network of cells with centers of hot, rising material and edges of cooler, sinking material. The rising material 
(moving towards the observer) is hotter, and so contributes more light to the spectrum, resulting in a convective blueshift. The velocities at different heights in the atmosphere, the different angles taken by our line of sight at different parts of the stellar disk, and the turbulent nature of the motions combine produce an asymmetric line profile, overall. Finally, the stochastic creation, destruction, and reorganization of these granules make this profile time-variable.

The amplitudes of these variations are of order meters per second, and decrease with increasing surface gravity Bastien et al $(2013,2014)$. These motions are also altered by the strength of the global surface field strength, resulting in RV variations on the timescales of stellar activity cycles (Lovis et al 2011).

Stars also undergo global oscillations excited by the surface granulation and magnetic events. These are probed by the Sun using helioseismology (and on stars via asteroseismology), and the dominant mode is the 5 minute p-mode oscillation. The up and down motions visibile on different parts of the stellar disk due to these oscillations do not precisely cancel, and the result is precise radial velocity variations on minutes-to-hours timescales with amplitudes of order $1 \mathrm{~m} / \mathrm{s}$. (Kjeldsen et al 2005)

\section{Identifying Jitter}

There are two broad strategies for dealing with jitter: avoidance and mitigation.

Avoidance is a viable strategy for surveys that can seek the "quietest" stars, especially those that require no more than $1 \mathrm{~m} / \mathrm{s}$ precision. In this case, bright, unevolved, old, late-G and K dwarfs offer the best opportunity to find low-amplitude planets with minimal jitter mitigation. (e.g. Wright 2005; Howard et al 2009).

Below $1 \mathrm{~m} / \mathrm{s}$, or for other kinds of stars, one must deal with jitter. The different kinds of jitter require different mitigation strategies. The two most common are to correlate radial velocities with activity indicators, such as emission in the cores of deep lines and photometry, and to carefully examine the shapes of lines for evidence of non-center-of-mass line shifts.

\section{Magnetic Activity Indicators}

Stellar magnetic activity results in strong, tangled field lines in stellar atmospheres, which heat the gas via magnetic reconnection and Alfvén waves. This creates the temperature inversion responsible for the existence of stellar chromospheres, which, being optically thin in the continuum, cool predominantly via emission in resonance lines such as CaII $\mathrm{H} \& \mathrm{~K}, \mathrm{NaI} \mathrm{D}$, and $\mathrm{H}-\alpha$. This emission appears in stellar spectra as a filling-in or inversion of the cores of these absorption features in cool stars.

The amount of emission in the cores of these lines gives an indication of the amount of cooling (and, therefore, heating) in the chromosphere, and so serves as a good proxy for the overall level of magnetic activity on the star (at least, on the hemisphere facing the Earth). 
Perhaps surprisingly, given the somewhat tenuous connection between the overall strength of the global field and the mechanisms by which that field creates spurious $\mathrm{RV}$ signatures, the measured strength of these emission features often has a simple relationship to the Doppler anomaly.

In the case of rotationally modulated spots, the Doppler signature is out of phase with the activity measurements by $\pi / 2$, because the spots are most prominent at the center of the disk, where they have zero rotational motion in the radial direction. In this case, photometry may also show a signal in-phase with the activity measurements (Queloz et al|2001a).

In the case of magnetic activity cycles and other variations in the global field strength, the effect is a simple correlation, with stronger fields yielding more redshifted lines. Neglecting to check for such a correlation can lead to spurious planet detections (Wright et al|2008, Robertson et al 2014). In some cases, this simple correlation is probably due to the suppression of convective blueshift as the field lines restrict the motion of the (slightly) ionized atmosphere (e.g. as in the case of $\alpha$ Cen B Dumusque et al 2012).

Even in the case where a direct correlation between activity indicators and RVs is not clear, the effects of stellar magnetic activity can be diagnosed via their rotational modulation. For sufficiently densely sampled activity time series, a power spectrum will often reveal the rotation period (and harmonics thereof). Any RV variation at these periods is suspect. Of course, real planets may have orbital periods that match these periods (as in the case of $\tau$ Boo $b$ Butler et al 1997), whether simply by coincidence or due to tidal locking, but in general the burden of proof for a claimed exoplanet discovery grows higher in the presence of such coincidences, especially for low-amplitude planets (Robertson and Mahadevan 2014).

\section{Line shape analysis}

Sources of spurious RV changes will, in general, not have exactly the same spectral signature as a Doppler shift due to true center-of-mass motion. While true Doppler shifts will leave line shapes unchanged, rotationally modulated spots or changes to the convective blueshift pattern will alter those shapes, if only slightly.

The most commonly seen measures of line shape are the line bisector-which traces the center a line as a function of depth below the continuum — and the width of the lines. True Doppler shifts will preserve these shapes, while changes in a line's profile should alter them (Hatzes 1996). The "span" of the bisector is the difference in the bisector position near the top and bottom of the line, measured in units of velocity. This is equivalent to the inverse of the mean slope of the bisector (for continuum normalized spectra), and is usually designated BIS (Queloz et al 2001a; Boisse et al 2011).

Correlations between measured radial velocities and BIS or line widths are thus red flags that the signal is not due to the reflex motion of an orbiting companion. It may indicate a spectrum blended with light from other stars, stellar pulsations, or other sources of jitter (see Wright et al 2013, for several examples.) 
Bisector and other line shape variations are measured most easily in stable spectrographs, where the shape of the cross-correlation function (CCF) can serve as a proxy for the mean shape of all lines used to derive velocities. They are not routinely measured in absorption cell spectroscopy.

\section{Wavelength Dependence}

True center-of-mass motion shifts all line wavelengths by the same fraction, while spurious effects will generally have different effects on lines of different wavelengths, depths, formation heights, and ionization states. For instance, rotationally modulated spot-induced RV anomalies should be less pronounced in the infrared, where brightness temperature contrasts are lower.

In principle, RVs measured from various lines should be checked for consistency, but in practice this is difficult. In absorption cell velocimetry, the spectrum is not decomposed into individual lines, and only a relatively narrow range of wavelengths is examined, so measured RVs cannot be examined as a function of wavelength or line properties. The problem is more tractable in stable spectrograph velocimetry, where individual lines are typically chosen for the analysis and can, in principle, be compared with each other for consistency.

\section{Target Selection}

The first targets of precise Doppler surveys were generally very bright (naked-eye) stars because early practitioners were generally using small telescopes, and because of the large number of photons needed to make a precise Doppler measurement. The stars must also have a rich set of absorption features to measure, making hot stars unsuitable targets, and must have narrow features, favoring stars with low projected rotational velocities $(v \sin i<10 \mathrm{~km} / \mathrm{s})$. Young stars have many surface features and

flares that make them unsuitable for the highest precision work, in addition to their high rotational velocities.

Giant stars also turn out to be poorer precise Doppler targets, because their atmospheres exhibit large variability in their Doppler motions (e.g. Hekker et al 2006). The highest precision is thus achieved on bright, old, dwarf, cool stars, which typically show intrinsic variations only at the $1 \mathrm{~m} / \mathrm{s}$ level (Wright 2005).

There are many reasons to push the technique outside of this optimal region. One, is exploration of the dependence of planet occurrence on stellar mass (e.g. Johnson et al 2010). Another is to measure the masses of planets known to transit, such as the faint, sometimes young or evolved targets of the Kepler and $K 2$ missions (Borucki et al 2010). 
Optical vs. Infrared

In general, precise Doppler work is most easily performed in the optical, where absorption cells are well calibrated, detectors are better behaved and more easily calibrated, and telluric absorption and emission is less difficult to work with. There are compelling reasons to pursue infrared precise Doppler work, however.

One is if one wishes to optimize not precision, but sensitivity to planets in the Habitable Zone (Kasting et al 1993), where surface liquid water is most likely to be found. Very cool late-M dwarfs have close-in Habitable Zones, and so these planets have shorter periods than around $\mathrm{G}$ and $\mathrm{K}$ dwarfs. This, combined with the more favorable planet-to-star mass ratios for a given planet mass, makes the necessary precision for discovering their Habitable-Zone planets an order of magnitude less stringent. Since these very cool stars have very little optical luminosity, the only practical solution is to measure their spectra in the near infrared where they have most of their energy output.

Recently, these and other concerns have created a strong push to extend precise Doppler velocimetry to the near infrared, with stable instruments such as the Habitable Zone Planet Finder and CARMENES (Mahadevan et al 2012, Quirrenbach et al 2010); and gas cell work as well (Bean et al 2010, Gao et al 2016).

\section{Future Challenges and Opportunities}

Precise radial velocimetry will continue to be a cornerstone of exoplanetary research into the foreseeable future. Space-based transit surveys are often limited by the availability of radial velocities to rule out many common sources of false positives, and to measure the masses of the planets discovered. Discovery of Jupiter analogs and very-long-period companions require decades of observation, making archival radial velocities relevant for years to come.

Advances in both instrumental precision and the understanding of the sources of and methods for mitigating stellar jitter will push radial velocity sensitivity down to lower and lower mass planets, with a common goal being the detections of an Earth analog (requiring detection of a $9 \mathrm{~cm} / \mathrm{s}$ signal). The most ambitious plans call for stability of $1 \mathrm{~cm} / \mathrm{s}$ with spectrographs that can access enough collecting area to ensure the measurements are not photon limited (e.g. CODEX Pepe and Lovis 2008).

\section{References}

Bastien FA, Stassun KG, Basri G Pepper J (2013) An observational correlation between stellar brightness variations and surface gravity. Nature500:427-430, DOI 10.1038/nature12419, 1308.4728 
Bastien FA, Stassun KG, Pepper J et al (2014) Radial Velocity Variations of Photometrically Quiet, Chromospherically Inactive Kepler Stars: A Link between RV Jitter and Photometric Flicker. AJ147:29, DOI 10.1088/0004-6256/147/2/29, 1310.7152

Bean JL, Seifahrt A, Hartman H et al (2010) The Proposed Giant Planet Orbiting VB 10 Does Not Exist. ApJ711:L19-L23, DOI 10.1088/2041-8205/711/1/L19

Boisse I, Bouchy F, Hébrard G et al (2011) Disentangling between stellar activity and planetary signals. A\&A528:A4, DOI 10.1051/0004-6361/201014354

Borucki WJ, Koch D, Basri G et al (2010) Kepler Planet-Detection Mission: Introduction and First Results. Science 327:977-, DOI 10.1126/science.1185402

Butler RP, Marcy GW, Williams E et al (1996) Attaining Doppler Precision of 3 M s-1. PASP108:500

Butler RP, Marcy GW, Williams E, Hauser H Shirts P (1997) Three New "51 Pegasi-Type" Planets. ApJ474:L115+

Campbell B Walker GAH (1979) Precision radial velocities with an absorption cell. PASP91:540545, DOI 10.1086/130535

Chubak C, Marcy G, Fischer DA et al (2012) Precise Radial Velocities of 2046 Nearby FGKM Stars and 131 Standards. ArXiv e-prints 1207.6212

Dumusque X, Pepe F, Lovis C et al (2012) An Earth-mass planet orbiting $\alpha$ Centauri B. Nature491:207-211, DOI 10.1038/nature11572

Fischer DA, Anglada-Escude G, Arriagada P et al (2016) State of the Field: Extreme Precision Radial Velocities. PASP128(6):066,001, DOI 10.1088/1538-3873/128/964/066001, 1602 . 07939

Gao P, Plavchan P, Gagné J et al (2016) Retrieval of Precise Radial Velocities from Near-infrared High-resolution Spectra of Low-mass Stars. PASP128(10):104,501, DOI 10.1088/1538-3873/ 128/968/104501, 1603.05997

Hatzes AP (1996) Simulations of Stellar Radial Velocity and Spectral Line Bisector Variations: I. Nonradial Pulsations. PASP108:839, DOI 10.1086/133805

Hekker S, Reffert S, Quirrenbach A et al (2006) Precise radial velocities of giant stars. I. Stable stars. A\&A454:943-949, DOI 10.1051/0004-6361:20064946, astro-ph/0604502

Howard AW, Johnson JA, Marcy GW et al (2009) The NASA-UC Eta-Earth Program. I. A SuperEarth Orbiting HD 7924. ApJ696:75-83, DOI 10.1088/0004-637X/696/1/75,0901.4394

Johnson JA, Aller KM, Howard AW Crepp JR (2010) Giant Planet Occurrence in the Stellar MassMetallicity Plane. PASP122:905-915, DOI 10.1086/655775, 1005.3084

Kasting JF, Whitmire DP Reynolds RT (1993) Habitable Zones around Main Sequence Stars. Icarus 101:108-128, DOI 10.1006/icar.1993.1010

Kjeldsen H, Bedding TR, Butler RP et al (2005) Solar-like Oscillations in $\alpha$ Centauri B. ApJ635:1281-1290, DOI 10.1086/497530

Latham DW, Stefanik RP, Mazeh T, Mayor M Burki G (1989) The unseen companion of HD114762 - A probable brown dwarf. Nature339:38-40, DOI 10.1038/339038a0

Lovis C, Dumusque X, Santos NC et al (2011) The HARPS search for southern extra-solar planets XXXI. Magnetic activity cycles in solar-type stars: statistics and impact on precise radial velocities. A\&A, submitted, arXiv:11075325 arXiv:1107.5325

Mahadevan S, Ramsey L, Bender C et al (2012) The habitable-zone planet finder: a stabilized fiber-fed NIR spectrograph for the Hobby-Eberly Telescope. In: Ground-based and Airborne Instrumentation for Astronomy IV, Proc SPIE, vol 8446, p 84461S, DOI 10.1117/12.926102, 1209.1686

Marcy GW Butler RP (1992) Precision radial velocities with an iodine absorption cell. PASP104:270-277, DOI 10.1086/132989

Mayor M Queloz D (1995) A Jupiter-Mass Companion to a Solar-Type Star. Nature378:355-+

Pepe FA Lovis C (2008) From HARPS to CODEX: exploring the limits of Doppler measurements. Physica Scripta Volume T 130(1):014007, DOI 10.1088/0031-8949/2008/T130/014007

Pepe FA, Cristiani S, Rebolo Lopez R et al (2010) ESPRESSO: the Echelle spectrograph for rocky exoplanets and stable spectroscopic observations. In: Ground-based and Airborne Instrumentation for Astronomy III, Proc SPIE, vol 7735, p 77350F, DOI 10.1117/12.857122 
Queloz D, Henry GW, Sivan JP et al (2001a) No planet for HD166435. A\&A379:279-287

Queloz D, Mayor M, Udry S et al (2001b) From CORALIE to HARPS. The way towards $1 \mathrm{~m} \mathrm{~s}^{-1}$ precision Doppler measurements. The Messenger 105:1-7

Quirrenbach A, Amado PJ, Mandel H et al (2010) CARMENES: Calar Alto high-resolution search for M dwarfs with exo-earths with a near-infrared Echelle spectrograph. In: Ground-based and Airborne Instrumentation for Astronomy III, Proc SPIE, vol 7735, p 773513, DOI 10.1117/12. 857777

Robertson P Mahadevan S (2014) Disentangling Planets and Stellar Activity for Gliese 667C. ApJ793:L24, DOI 10.1088/2041-8205/793/2/L24, 1409.0021

Robertson P, Mahadevan S, Endl M Roy A (2014) Stellar activity masquerading as planets in the habitable zone of the M dwarf Gliese 581. Science 345:440-444, DOI 10.1126/science. $1253253,1407.1049$

Wright JT (2005) Radial Velocity Jitter in Stars from the California and Carnegie Planet Search at Keck Observatory. PASP117:657-664, DOI 10.1086/430369

Wright JT Eastman JD (2014) Barycentric Corrections at $1 \mathrm{~cm} \mathrm{~s}^{-1}$ for Precise Doppler Velocities. PASP126:838-852, DOI 10.1086/678541, 1409.4774

Wright JT Gaudi BS (2013) Exoplanet Detection Methods, p 489. DOI 10.1007/ 978-94-007-5606-9_10

Wright JT Howard AW (2009) Efficient Fitting of Multiplanet Keplerian Models to Radial Velocity and Astrometry Data. ApJS182:205-215, DOI 10.1088/0067-0049/182/1/205,0904.3725

Wright JT, Marcy GW, Butler RP et al (2008) The Jupiter Twin HD 154345b. ApJ683:L63-L66, DOI 10.1086/587461, arXiv:0802.1731

Wright JT, Roy A, Mahadevan S et al (2013) MARVELS-1: A Face-on Double-lined Binary Star Masquerading as a Resonant Planetary System and Consideration of Rare False Positives in Radial Velocity Planet Searches. ApJ770:119, DOI 10.1088/0004-637X/770/2/119, 1305.0280 